\title{
ABRIENDO NUEVOS ESPACIOS ACADÉMICOS Y CIENTÍFICOS: PRIMERAS EDUCADORAS EN LA UNIVERSIDAD PEDAGÓGICAY TECNOLÓGICA DE COLOMBIA $^{1}$
}

\author{
Mgs. Ana Hercilia Hamón Naranjo ${ }^{2}$ \\ Doctorado en Ciencias de la Educación \\ RUDECOLOMBIA \\ Grupo de Investigación HISULA \\ anahamon20@yahoo.es
}

Recepción: 05/10/2009

Evaluación: 08/10/2009

Aceptación: 29/10/2009

Articulo de Reflexión

\begin{abstract}
RESUMEN
Da a conocer la forma de ingreso, el perfil profesional y el trabajo académico de las primeras docentes de la Universidad Pedagógica y Tecnológica de Colombia (UPTC). Aspectos como el origen, la familia y los estudios de las primeras docentes son analizados, así como el sistema de nombramientos. El artículo es producto de una investigación sustentada en la corriente historiográfica de la historia social de la educación y complementada con el método comparativo. Se concluye que no fue fácil la llegada de la mujer a la docencia en la UPTC y que tal llegada constituyó un hecho transcendental en la institución. Las primeras docentes de la UPTC fueron profesionales de familias destacadas de Boyacá; al final se destaca a la docente María Teresa León
\end{abstract}

\footnotetext{
Artículo presentado en el Simposio Educadores Latinoamericanos, en el marco del III Seminario Taller Internacional Vendimia "La Construcción de la nación en Latinoamerica, siglos XIX al XXI: comparaciones y conexiones", Villa de Leyva (Boyacá), noviembre 11, 12 y 13 de 2009.

2 Doctoranda del Programa en Ciencias de la Educación, de la Universidad Pedagógica y Tecnológica de Colombia - RUDECOLOMBIA, Administradora de Empresas, Especialista en Finanzas, Alta Gerencia en Mercadotecnia y Magíster en Desarrollo Rural, miembro del Grupo de Investigación "Historia y Prospectiva", de la Universidad Latinoamericana HISULA.
} 
Ana Hamón Naranjo

Leal, a quien le correspondió abrir el espacio académico y científico para la mujer en la UPTC, con resultados de cambios sociales e institucionales.

Palabras clave: Historia, Educadoras, Universidad, Academia, Ingreso, Perfil.

\title{
OPENING NEW ACADEMIC AND SCIENTIFIC SPACES: THE FIRST FEMALE PROFESSORS AT UNIVERSIDAD PEDAGÓGICA Y TECNOLÓGICA DE COLOMBIA
}

\author{
Mgs. Ana Hercilia Hamón Naranjo \\ Doctorado en Ciencias de la Educación \\ RUDECOLOMBIA \\ Grupo de Investigación HISULA \\ anahamon20@yahoo.es
}

\begin{abstract}
In the fifties, female educator is the big absent agent from the Colombian university, where the full teaching faculty was made by merely male educators. It was precisely in the sixties when the first female professors started teaching at the Universidad Pedagógica y Tecnológica de Colombia, and slowly the number of them increased in the seventies. This article analyses some aspects such as origin, family and studies that allow to know briefly something about the first women who joined the UPTC's teaching faculty. It is worth to emphasize that professor María Teresa León Leal was the one who opened the academic and scientific space to women in this teaching cloister.
\end{abstract}

Key words: History, teachers, university, academic, to enter, profile. 


\section{INTRODUCCIÓN}

En sus inicios la UPTC (sección masculina de la Universidad Pedagógica de Colombia) se caracterizó por tener solo presencia masculina, tanto de profesores como de estudiantes, y por ser uniprofesional, alrededor de la Facultad de Educación, con los programas de Filología e Idiomas, Psicopedagogía, Filosofía, Biología y Química, Matemáticas y Física y Ciencias Sociales y Económicas. La Facultad propugnaba ser luz de la educación colombiana e hispanoamericana, para la formación de los dirigentes de la educación, como rectores y supervisores, y de los profesores de las Escuelas Normales y de educación media en las diversas áreas. Posteriormente, la institución pasa a ser una universidad multiprofesional, descentralizada y con presencia de hombres y mujeres en los estamentos estudiantil y profesoral, en las distintas facultades y programas académicos.

El ingreso de las mujeres docentes a la UPTC fue tardío; sólo se logró a partir del año 1961, mucho después de que lo habían permitido otras universidades, como la Nacional, la del Cauca, la de Antioquia y la de Cartagena; treinta años después de la llegada de Paulina Beregoff a la Universidad de Cartagena, en 1921, como docente en bacteriología y parasitología, e investigadora de enfermedades tropicales como la fiebre amarilla. Se consolida su ingreso a partir de la llegada de la maestra María Teresa León Leal a partir del 15 de marzo de 1965 quien le correspondió abrir las puertas de la universidad en los diferentes desempeños académicos. El aporte de este trabajo es demostrar que la mujer participa activamente en la vida de la universidad y que realiza una labor significativa, pero que no es visible ante la comunidad académica y científica nacional ni regional, por falta de estudios que permitan conocer su labor.

La metodología utilizada en el desarrollo de la investigación se sustenta dentro de la corriente historiográfica de la "historia social de la educación"3 y de las mentalidades, que permite el análisis y la interpretación histórica de las mujeres en la universidad colombiana, estableciendo los contextos políticos nacionales, de élites con una mentalidad y una ideología presente en la cultura masculina, que impedía el desarrollo de la mujer en la universidad. Igualmente este estudio no tendría relevancia sin el método comparativo que tiene como objetivo "lograr la identificación de diferencias o semejanzas con respecto a la aparición de un evento en dos o más contextos." Por tal motivo, la metodología de la educación comparada está presente en el análisis de la investigación.

\footnotetext{
3 Historia social de la educación es el estudio de las interdependencias entre factores figurativos de las estructuras históricos sociales (demográficas, económicas, sociales, ideológicas, políticas y mentales, etc.) y el sistema escolar que dichas estructuras vehiculan entendiendo por sistema escolar toda manifestación educativa que se dé en una determinada sociedad. En: CARDOSO, Ciro. (1997): Los métodos de la historia, México, Editorial Grijalbo, p. 74.

4 http://ares.unimet.edu.ve/didactica/
} 


\section{La educadora universitaria, la gran ausente en la década de los cincuenta}

En la década de los cincuenta, la mujer educadora es la gran ausente en la universidad colombiana. Por ejemplo, en la Universidad Pedagógica, en este periodo, "la totalidad de sus docentes, 51, eran varones," la nómina de docentes, en su totalidad, pertenece a la única Facultad existente, la de Educación, y estaba distribuida en los siguientes programas: Filología e Idiomas, 13 profesores; Ciencias Sociales, 11; Educación, 11; Biología y Química, 8; Matemáticas y Física, 6; Especialización, 2. La mayoría de docentes fueron alumnos o docentes de la Normal Masculina, aspecto que facilitó el ingreso o el ascenso de los profesores a la Universidad, por los lazos de amistad o relación académica de las dos instituciones.

Es relevante recordar que la UPTC tiene sus orígenes en la Escuela Normal Superior, que era masculina. El hecho de haber trasladado la Escuela Normal Superior de Tunja a Bogotá (1935) y posteriormente traer a Tunja la sección masculina (1952), y establecer en Bogotá la sección femenina, fue un aspecto que limitó el ingreso de las docentes a la Universidad, como lo manifestó en su momento la Dra. Francisca Radke: "las señoritas no van a educar muchachos," sino que educarán niñas, dentro de principios morales y orientación religiosa. Lo anterior refleja la influencia sobre la mujer de la cultura machista y la religión católica, sustentada en el Partido Conservador y en algunos liberales en el Congreso, contraria al avance de los derechos de la mujer en el siglo XX. Como lo indica el doctor Ocampo, "debemos anotar que mediante Decreto 547 del 23 de febrero de 1954 se creó la Universidad Pedagógica Femenina en Bogotá, independizándola de la Universidad Pedagógica de Colombia."7

En esta separación de la sección femenina influyó la Dra. Radke, como se puede evidenciar en las actas del Consejo Directivo; ${ }^{8}$ ella nunca estuvo de acuerdo con que estudiaran en el mismo centro educativo hombres y mujeres, por lo que siempre solicitó su separación. La educación mixta era interpretada por la doctora Radke ${ }^{9}$ y la Iglesia como generadora de indisciplina y desorden, aunque esto con el tiempo se desmintió, al demostrarse que las mujeres en la universidad, en todo momento, tuvieron orden, respeto y buen comportamiento.

Después de una larga lucha de las jóvenes y de padres de familia, como se aprecia en una carta con varias firmas, dirigida al señor rector, en donde le solicitan "permitir desde el año próximo venidero el ingreso de señoritas a las Facultades de la Universidad

\footnotetext{
5 Archivo Histórico, Universidad Pedagógica y Tecnológica de Colombia 1960-1980.

6 Archivo Histórico, Universidad Pedagógica y Tecnológica de Colombia, Acta $\mathrm{N}^{\mathrm{o}} 7$ de 1954, Consejo Directivo, legajo $\mathrm{N}^{\circ} 2$.

7 OCAMPO LÓPEZ, Javier. (1978): Educación, Humanismo y Ciencia, Tunja, Ediciones La Rana y el Águila, p. 209.

8 Archivo Histórico, Universidad Pedagógica y Tecnológica de Colombia, Asuntos varios rectoría, Acta Consejo Directivo No.4 de 1957

9 Ibídem, p. 2.
}

Rhela. Vol. 13. Año 2009, pp. $28-46$ 
Pedagógica en calidad de externas, para que bajo su orientación y sabiduría logren los mismos fines que persiguen y obtienen los jóvenes varones que han sido confiados a su cargo," ${ }^{10}$ este deseo se cumple, en el año 1957; más adelante, también se facilita el ingreso de mujeres profesoras a las aulas universitarias.

En esta época, la influencia de la Iglesia era fundamental en las decisiones de la Universidad, además de que se encontraban sacerdotes dictando clases en la Facultad de Educación; también en el Consejo Directivo hacía presencia con el "Representante del Eminentísimo Señor Cardenal;""11 lo anterior era determinante en la preservación de los principios morales y la dirección espiritual de la institución, uno de los factores que también contribuyeron al fortalecimiento de la presencia del machismo y liderazgo del partido conservador y a la vez, dificultó la llegada de las mujeres a la Universidad, aunque a nivel nacional e internacional en varias universidades ellas ya habían ingresado.

\section{Primeras Educadoras en la Universidad Pedagógica y Tecnológica de Colombia}

Sistema de nombramientos y perfil profesional de las primeras educadoras de la UPTC. El sistema de nombramiento de docentes en la Universidad Pedagógica y Tecnológica de Colombia, durante el periodo de investigación, se enmarca dentro de las principales normas del momento, como son la Ley 73 del 30 de diciembre de 1958, la Ley 73 del 14 de diciembre de 1973 y los Acuerdos 001 del 4 de enero de 1961, 003 del 14 de enero de 1961, 26 del 12 de noviembre de 1964 y 18 del 29 de diciembre de 1967; a partir de estas leyes y acuerdos se expiden las respectivas resoluciones de nombramiento, que más adelante, en el desarrollo del artículo, se analizan detalladamente y que rigen o se aplican en igualdad de condiciones para hombres y mujeres. Como se puede observar, las anteriores normas corresponden a la época de Universidad Pedagógica y a los inicios de Universidad Pedagógica y Tecnológica de Colombia.

Es importante destacar la organización tan minuciosa y extraordinaria de la UPTC en cuanto a profesores, como manifiesta la doctora Lina Parra: "empezando por los nombramientos ya fuera de tiempo completo o por contrato, se hacían por Resolución Rectoral, allí se nombraban desde decanos hasta profesores auxiliares, cada uno con una función específica; generalmente los decanos y altos cargos de las facultades estaban en poder de los profesores," 12 estos nombramientos también se hacían por resolución rectoral, es decir, de la misma forma que las demás universidades nacionales.

\footnotetext{
${ }^{10}$ Archivo Histórico, Universidad Pedagógica y Tecnológica de Colombia, Asuntos varios rectoría, junio 1955 .

11 Archivo Histórico, Universidad Pedagógica y Tecnológica de Colombia, Acta $\mathrm{N}^{\circ} 5$, Consejo Directivo Carpeta $\mathrm{N}^{\circ} 6$ de 22 de marzo de 1957.

12 PARRA BÁEZ, Lina Adriana. (2006): La formación de maestras universitarias colombianas entre 1950-1960, Tunja, Tesis de grado para optar el título de doctor en Ciencias de la Educación, Universidad Pedagógica y Tecnológica de Colombia - RUDECOLOMBIA, p. 212.
} 
Costumbres que hacen parte de la historia de los primeros nombramientos de educadoras en la Universidad Pedagógica y Tecnológica de Colombia. Son varias las costumbres que hacen parte de la historia de la vinculación de las primeras docentes en la Universidad Pedagógica y Tecnológica de Colombia; a continuación se describen las más frecuentes:

- Las docentes cuentan con algún pariente ya vinculado como docente en la universidad: "hermanos, esposo, tío, primo, etc,"13 que les informa sobre las necesidades de servicios académicos, orientan en el proceso, apoyan y facilitan su ingreso.

- En otros casos, por haber "realizado su carrera profesional en la UPTC, donde establecieron vínculos de amistad con sus profesores, directivos y además demostraron ser excelentes profesionales"14 $\mathrm{y}$ al momento del requerimiento de un servicio son las primeras en ser invitadas a suplir esa necesidad.

- Por amigos o compañeros de estudios que trabajan como docentes en la UPTC, "me informó que había la vacante, que estuviera pendiente." ${ }^{15}$ Aquí el papel de las referencias personales, que funcionan mucho en nuestro medio y mucho más cuando es de alguien con cierto tipo de reconocimiento y prestigio académico, se respeta y se cumple.

- Por influencia de alguna persona importante del ámbito nacional, principalmente de los Ministerios, como Educación y Hacienda, quien presenta la hoja de vida al Rector y este procede a nombrar al docente: "un amigo del Ministerio de Educación presentó mi hoja de vida al Rector." ${ }^{16}$

- Presentación de directivos de la Universidad Pedagógica y Tecnológica de Colombia, llámese Consejo Superior, Consejo Académico o Rector: "me avisó una persona que estaba en el Consejo Superior de la Universidad que había una vacante en economía, me invitó a que me presentara al concurso, así lo hice."17

- Otra forma común de vinculación fue a través de la Normal de Varones; en primer lugar, por ser una institución anexa a la Universidad y, en segundo lugar, por su trayectoria: "al comienzo la mayoría de docentes de la Universidad que se vinculaban venían de la Normal de Varones de Tunja." 18

- Igualmente, se vincularon a través del Instituto Técnico Rafael Reyes ${ }^{19}$ de Duitama, institución anexa a la Universidad; en este Instituto laboraron varias docentes que más tarde fueron trasladas y nombradas en la UPTC.

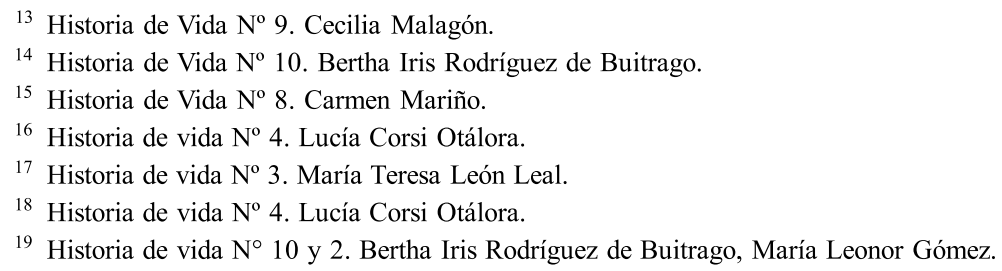


- Al final del periodo de estudio se localiza otro medio de vinculación fue a través de convocatoria pública realizada en periódicos, principalmente El Tiempo (año 1978); en este caso, previamente alguien que estaba dentro de la Universidad le informaba al aspirante que estuviera pendiente del periódico para que conociera la convocatoria y se pudiera presentar.

Es necesario tener presente que en esa época no era tan complicada la vinculación como docente a la Universidad; por el contrario, había varias opciones para elegir la institución; una de las razones de la baja oferta laboral a este nivel era que poca gente podía acceder a la educación superior; en muchos casos, esta educación era elitista y para unos pocos privilegiados. Por otra parte, era una sociedad rural, (para la década del sesenta estaba alrededor del $48 \%$ y en la década del setenta del $40 \%)^{20}$ donde no había mucho interés por trabajar fuera de su hogar, en razón a la existencia de una economía campesina de subsistencia y a los bajos niveles educativos. Además, no existían en la provincia instituciones educativas del nivel superior que brindaran la oportunidad a las mujeres de estudiar, lo que las obligaba a trasladarse a Bogotá. Aquí es importante recordar que la mujer en esta época debía permanecer al lado de su familia, por lo tanto eran pocas las que tomaban la decisión de desplazarse a la ciudad a estudiar en la universidad.

\section{Perfil de las Primeras Educadoras que Ingresan a la UPTC}

En este apartado se analizan algunos aspectos como origen, familia y estudios, que permiten conocer, en forma general, quiénes fueron las docentes que se vincularon a la UPTC en el periodo de estudio. Entre los principales lugares de origen de las primeras docentes se encuentran, en primer lugar, municipios de Boyacá, entre los cuales sobresalen: San José de Pare, Cocuy, El Espino, Chiquinquirá, Floresta y Tunja; en segundo lugar, la ciudad de Bogotá; en tercer lugar, la ciudad de Bucaramanga, y, en mínima cantidad, otros municipios de Colombia.

Como ellas mismas lo manifestaron en las historias de vida, provenían de familias destacadas social y económicamente, indistintamente de la profesión u oficio de sus progenitores, como por ejemplo: Secretarios de Educación Departamental, supervisores, docentes de primaria y secundaria, abogados y comerciantes. En lo referente a los hermanos, realmente eran familias pequeñas; en la mayoría oscilaban entre tres y, máximo, seis hermanos; la mayoría de ellos se iban a estudiar a Bogotá y algunas de ellas debían quedarse en Tunja, para realizar su carrera profesional.

Las docentes que se incorporaron en esta época eran jóvenes; sus edades oscilaban entre 21 y 32 años; en cuanto a su estado civil, la gran mayoría eran solteras; las pocas docentes casadas tienen en su mayoría entre dos y tres hijos, lo que expresa que para esta época ya comenzaba a presentarse una reducción de estos; ellas tienen mayor o

\footnotetext{
${ }^{20}$ Hoja de Vida. Ibídem.
} 
igual desarrollo académico que sus esposos y predominaban los cónyuges profesores de la UPTC; otros son funcionarios de instituciones del gobierno en los ámbitos nacional y departamental, y unos pocos, trabajadores independientes.

En el tema de estudios se hace referencia a los secundarios y universitarios, en razón de la importancia en la toma de decisiones para elegir una carrera profesional y, por consiguiente, definir su desempeño laboral. A continuación se relacionan algunos de los principales colegios de secundaria donde ellas realizaron sus estudios: Colegio de Boyacá; Colegio de la Presentación, en Tunja y Bogotá; colegios públicos de Bucaramanga; Instituto Pedagógico de Bogotá y varias Escuelas Normales, entre ellas: Villa de Leyva, Sor Josefa del Castillo, de Chiquinquirá, Varones de Tunja. "Esta formación pedagógica fue trascendental para el ejercicio de la docencia, que más tarde la complementaban con los estudios universitarios." ${ }^{21}$

Entre las Instituciones de Educación Superior de mayor demanda por las mujeres se encuentran la Universidad Javeriana, al respecto se encuentra el siguiente testimonio: "Mientras estudiaba en la Javeriana en Bogotá, vivía donde un tío, quien me cuidada,"22 y la Universidad Pedagógica de Bogotá: "estudie en Bogotá porque en esa época no habían estudios superiores para mujeres en Tunja, vivía en una residencias de monjas." 23 Hay que destacar que todavía en esta época las mujeres no podían vivir solas; siempre estaban bajo la responsabilidad de algún familiar o en un internado, bien fuera de mojas o en las Normales. Entre otras universidades se encuentra la Nacional en Bogotá y la Industrial de Santander, de donde son egresadas un alto número de profesoras vinculadas hoy con universidades públicas, situación que favoreció en estas instituciones la influencia de izquierda y de movimientos de pensamiento socialista que predominaban en la época y que, de una u otra manera, influyeron en la UPTC, como se evidencia en los frecuentes paros y protestas.

En la década de los setenta comienzan a incorporase a la UPTC como docentes las primeras egresadas de esta; desearon estudiar en Bogotá, pero encontraron cierta oposición en las familias: "quería estudiar en Bogotá arquitectura pero mi mamá no me dejó, por tener que irme sola para una ciudad tan grande, y ella no aceptaba eso en esa época; por eso me tocó quedarme a estudiar en Tunja." ${ }^{24}$ Otra egresada de la UPTC nos comenta: "también tuve que estudiar en Tunja porque aquí vivían mis padres y debía estar con ellos, pero a mis hermanos sí los llevaron a estudiar en Bogotá." 25

Otra variable para tener en cuenta es la escogencia de la profesión; como las carreras que predominan en esta época son las licenciaturas, ellas las eligen porque piensan "que tienen más salida y posibilidades de trabajo;"26 existía una inmensa mayoría

\footnotetext{
${ }^{21}$ Historia de vida $\mathrm{N}^{\circ}$ 9. Cecilia Malagón.

${ }^{22}$ Historia de vida $\mathrm{N}^{\circ}$ 9. Cecilia Malagón.

${ }^{23}$ Historia de vida $\mathrm{N}^{\circ} 4$. Lucía Corsi Otálora.

${ }^{24}$ Historia de vida $\mathrm{N}^{\circ} 10$. Bertha Iris Rodríguez de Buitrago.

${ }^{25}$ Historia de vida $N^{\circ} 7$. Nohora Ruíz.

${ }^{26}$ Historia de vida $\mathrm{N}^{\circ}$ 4. Lucía Corsi Otálora.
} 
que desde que iniciaba la secundaria ya se decidía por la parte pedagógica, algunas en normales o institutos pedagógicos; algunas lo hacían porque veían que era una opción segura de trabajo, otras, por necesidad, en razón a que en cualquier momento debían responder por la familia, y en el magisterio no había problemas de empleo; otras, por tener que ir a estudiar fuera de su ciudad natal, y debían hacerlo internas, y las normales eran las instituciones que más brindaban estos internados; otras porque sus padres o algún pariente les aconsejaba la docencia. En conclusión, la docencia es una de las alternativas profesionales más preferidas por las mujeres, y coincide con el pensamiento de varios autores que estudian el tema de género, que expresan la continuidad del rol del trabajo del hogar al trabajo profesional; donde ellas trasladan los roles de la educación y el cuidado a lo público; "se propusieron unas áreas de desempeño en el medio universitario, tales como la educación, enfermería, bellas artes, que en nada diferían de lo que se venía ofreciendo tradicionalmente en el plano educativo a la mujer colombiana." 27

\section{Primera Educadora Nombrada por Resolución del Consejo Académico de la UPTC}

La primera mujer docente vinculada a la Universidad Pedagógica y Tecnológica de Colombia fue la licenciada Lucía Corsi Otálora, ${ }^{28}$ mediante Resolución del Consejo Académico número 025, de marzo 21 de 1961; en esta se lee: "designase a la señorita LUCÍA CORSI OTÁLORA, profesora auxiliar para el Departamento de Filología e Idiomas de la Facultad de Educación, a partir del 19 de enero del presente año, con una asignación mensual de \$1.620.0o;" 29 esta primera docente se ubica dentro de la caracterización del ingreso a partir de amistades y familiares dentro de la institución, en este caso su hermano Luis Corsi Otálora, profesor de la Facultad de Ingeniería, y el rector, que era amigo personal de la familia Corsi. ${ }^{30}$ Para su nombramiento, ella presentó título de Licenciada en Filología e Idiomas, de la Universidad Pedagógica Nacional, y diplomas de estudios en pronunciación, del Instituto Fhonetique de La Sorbona de París, realizados en el año 1958.

\footnotetext{
27 PIÑERES DE LA OSSA, Dora. (2004): Historia de la Universidad de Cartagena. Reforma y Modernidad 1920 - 1946, Tunja, Tesis de grado, Doctorado en Ciencias de la Educación. Universidad Pedagógica y Tecnológica de Colombia. RUDECOLOMBIA, p. 140

${ }^{28}$ CORSI OTÁLORA, Lucía. Nació en Tunja en el año 1933. Realizó sus estudios de primaria en el Colegio Selección de Tunja, que era de modalidad mixta; sus estudios secundarios, en el Colegio de Boyacá, y los estudios superiores los realizó en la Universidad Pedagógica de Bogotá. Durante esa época de estudiante vivía en unas residencias de Monjas. Viajó a París, donde complementó sus estudios de fonética y gramática, en el Instituto Fhonetique y en la Universidad de la Sorbona. Una vez realizados sus estudios en el exterior, regresó a Tunja y se vinculó como docente a la Universidad Pedagógica y Tecnológica de Colombia. En: historia de vida $\mathrm{N}^{\circ} 4$.

${ }^{29}$ ARCHIVO HISTÓRICO, Universidad Pedagógica y Tecnológica de Colombia, Rectoría. Resoluciones 1960-1961.

${ }^{30}$ Firma esta Resolución el Doctor Rafael Azula Barrera, Rector, quien era amigo personal de la familia.
} 
La Resolución de nombramiento de la primera mujer docente contiene un artículo único en el que dice: Hacer la designación con carácter de Interinidad (mayúscula y resaltado) en la Universidad Pedagógica y Tecnológica de Colombia y solo para la vigencia 1961. La fecha de esta resolución es el inicio del primer periodo de nombramientos de mujeres en la universidad, por lo tanto, marca el comienzo de una nueva etapa de la vida universitaria, es decir, pasar de una institución de nómina docente exclusivamente masculina a una mixta. Esta mujer abre las puertas de la universidad a las docentes, aunque fue una tarea difícil, crea un nuevo escenario, que ofrece otra alternativa profesional a las mujeres que han logrado superarse y han realizado grandes esfuerzos y luchas para romper costumbres hasta ahora arraigadas en nuestra sociedad.

El hecho de que Lucía Corsi (retrato 1), haya realizado una carrera profesional fuera de su ciudad natal (Tunja), en la Universidad Pedagógica Nacional de Bogotá, que le otorgó el título de licenciada en Filología e Idiomas, marca una nueva época para las mujeres, y más cuando debe coger vuelo de avión sola y atravesar el Atlántico para llegar y moverse en el continente europeo, cuyo desarrollo avanzado en todo sentido le hace ejercer todas sus habilidades humanas para salir adelante, y esto la hace fuerte para romper costumbres, demostrar su capacidad, su inteligencia y enfrentar debates, como lo hizo en la UPTC, una vez regresa de Francia y se vincula a la Universidad; todo lo anterior creó un ambiente favorable para que en los siguientes años llegaran más mujeres docentes a la institución.

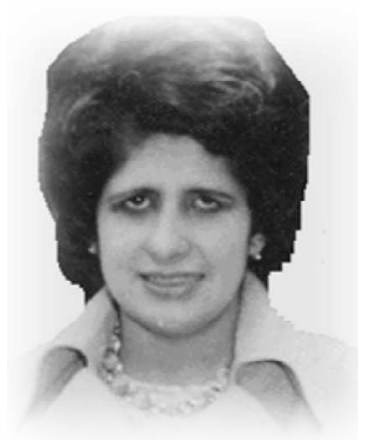

Retrato $\mathbf{N}^{\circ}$ 1. LUCÍA CORSI OTÁLORA. Primera Educadora por contrato Facultad de Educación, 1961

Universidad Pedagógica y Tecnológica de Colombia

Paralelo al nombramiento de Lucía Corsi como docente por contrato en la Universidad, a nivel nacional se presenta un hecho importante en la historia de la mujer colombina: la designación de Esmeralda Arboleda de Cuevas como Ministra de Comunicaciones; este suceso contribuye a romper barreras y a facilitar el ingreso de la mujer a la universidad.

Por otra parte, es importante señalar que la Ley 73 del 14 de diciembre de 1962, en su artículo 8, estableció los integrantes del Consejo Superior de la Universidad: "formarán parte del Consejo Superior Universitario, además de las personas anunciadas en el artículo 25 de la ley 73 de 1958, el señor Obispo de la Diócesis de Tunja o un delegado suyo y el señor gerente de la Empresa Siderúrgica Paz de Río". A pesar de la participación de la

Iglesia en el gobierno de la Universidad, no se encontraron en ningún documento evidencias de sus intervenciones; se tiene sí, conocimiento de su papel en el cumplimiento de los principios morales y éticos de los universitarios, aspecto que en alguna medida limitó el ingreso de la mujer a la Universidad.

\begin{tabular}{l|l} 
& Rhela. Vol. 12. Año 2009, pp. 28-46
\end{tabular}




\section{Paros y Protestas Estudiantiles por el Nombramiento de Educadoras en la Universidad Pedagógica y Tecnológica de Colombia}

La estadía en la Universidad de la licenciada Lucía Corsi fue fugaz, en razón a "protestas de los estudiantes" ${ }^{\prime 31}$ y aun de algunos profesores, a pesar de ser su hermano docente de ingeniería, lo cual representaba un gran respaldo para ella; pero no pudo continuar porque la situación se complicó y decidió abandonar la Universidad e irse a realizar una especialización en la ciudad Bogotá; sin embargo, sin duda alguna, este fue el principio del camino para muchas mujeres que esperaban una oportunidad en la Universidad.

Las protestas estudiantiles son definidas por Mauricio Archila como "conjunto de acciones sociales colectivas que expresan intencionalmente demandas o presionan soluciones ante el Estado, las entidades privadas o los individuos." ${ }^{32}$ Aquí los alumnos lideran acciones colectivas voluntarias para enfrentar la defensa de sus intereses estudiantiles, y arremeten contra las docentes, amparándose en la defensa de sus derechos.

Otra demostración de lo difícil y complicado que fue la vinculación o llegada de las mujeres como docentes a la Universidad Pedagógica y Tecnológica de Colombia fue la situación que vivió la señora Emma Rosas de Ospina, evidencia presentada en el acta n. ${ }^{\circ}$ 13 de 3 de abril de 1963 del Honorable Consejo Académico, en el numeral número tres: "enseguida pidió la palabra la señora Emma Rosas de Ospina, directora de la sección femenina de la Universidad, quien hizo una exposición alrededor del problema planteado por su nombramiento como profesora de la Facultad de Educación, en la Especialización de Filología e Idiomas, Sociales, Económicas y Biología, por haberse presentado allí un paro colectivo, como puede comprobarlo en las fallas que aparecen en los respectivos diarios de clase" ${ }^{\prime 33}$. La profesora Emma Rosa llegaba muy cumplida al aula correspondiente a dictar sus clases, pero los estudiantes no entraban al salón porque, según el estudiante integrante del Consejo Académicos, "no la aceptamos como profesora por diferentes motivos, entre ellos, falta de idoneidad y capacidad para dictar las clases, y otros motivos que ya fueron presentados al señor Rector mediante memorial y que es la expresión de la Asamblea General de estudiantes" ${ }^{\prime 34}$. La profesora manifiesta tener la capacidad intelectual para dictar las clases y solicita a los estudiantes le permitan ser escuchada para que puedan opinar al respecto, pero esto agravó la situación, hasta llegar al punto extremo de recibir la profesora ofensas personales y amenazas.

\footnotetext{
${ }^{31}$ Protestas estudiantiles en Colombia. Durante el final de los años cincuenta y en los decenios de los sesenta y setenta se fundaron numerosas organizaciones proclamadas revolucionarias, varias de las cuales promovieron sus propias guerrillas. La agitación estudiantil adquirió una frecuencia e intensidad inusitadas. Los estudiantes se sublevaron contra el despotismo imperante en los claustros, y cuando se desencadenó la represión oficial, lejos de amainar, el motín arreció. Extraído desde internet (online) el 23/04/2008 http:// www.juventudpatriotica.com/comunidad/mov71/doc2.pdf.

32 ARCHILA, Mauricio. (2001): Movimientos sociales, Estado y Democracia en Colombia, Bogotá, Litocamargo Ltda, p. 18.

33 ARCHIVO HISTÓRICO, Universidad Pedagógica y Tecnológica de Colombia, Rectoría, Acta del Consejo Académico N. ${ }^{\circ} 13$ del 3 de abril de 1963.

${ }^{34}$ Ibídem, p. 2.
} 
Los integrantes del Consejo Académico solicitan respeto por la profesora y le manifiestan su apoyo y respaldo; además indican que ella ha sido una excelente Directora de la Sección Femenina de la Universidad, y que también demostró su profesionalismo como Directora del Colegio Boyacá; pero los estudiantes en ningún momento permitieron que les dictara las clases, por lo tanto, más adelante, el Consejo Académico tuvo la necesidad de tomar otra decisión para no afectar el orden académico de la institución. Lo anterior demuestra que el ingreso de las mujeres docentes a la Universidad Pedagógica y Tecnológica de Colombia no fue un camino fácil, por el contrario, fue necesario el sacrificio de las primeras docentes que lo intentaron; pero, al mismo tiempo, ellas abrieron el debate y pusieron en el ambiente académico la necesidad de abrir espacios, ya que no había ningún impedimento para hacer lo contrario; en esta fecha ya habían ingresado varias mujeres docentes a otras universidades, como la Nacional y la de Antioquia. Lo anterior confirma la marcada tendencia tradicional y machista de nuestra sociedad boyacense respecto al papel de la mujer en la sociedad.

\section{Procedimiento para el Nombramiento de Docentes}

En los archivos institucionales se evidenció, en primer lugar, la solicitud de docentes que hacen los respectivos Jefes de Departamento a los Decanos, y estos la presentan ante el Consejo de Facultad, que la estudia y, según la necesidad académica, hace la solicitud de nombramiento al Consejo Académico. Lo anterior se pude apreciar en el oficio de fecha 24 de enero de 1963, firmado por el profesor Jesús M. Obando Marín, Jefe del Departamento de Lenguas, en donde expresa: "me permito presentar a usted la relación de profesores de tiempo completo que se necesitan para el desarrollo y cumplimiento de las actividades académicas en la Sección de Filología e Idiomas y en el Departamento de Lenguas, en el año lectivo de 1963, para los fines de su nombramiento en el Consejo Académico de la Universidad Pedagógica y Tecnológica de Colombia." ${ }^{35}$

En esta relación también se establecen las materias que tendrá a su cargo cada profesor y las horas de actividad docente; en la carta anteriormente citada se solicita los servicios de ocho docentes, entre ellos se encuentra el nombre de la licenciada Lucía Corsi Otálora (Profesora Auxiliar), Lengua Francesa 1, 2, 3 y 4, Sección de Filología e Idiomas: 14 horas. Se evidencia en esta relación que el menor número de horas asignadas corresponde a la profesora Lucía Corsi. Una vez cumplido el trámite anterior, el Consejo Académico una vez realizado el respectivo estudio, resuelve ordenar el nombramiento.

\section{Se abren definitivamente las puertas a las docentes}

Una vez superada la crisis presentada en los años anteriores por el nombramiento de las primeras mujeres docentes, y después de cinco años, se nombra de nuevo, por Resolución Rectoral número 46 de 1965, a una mujer como profesora, y en este caso de planta: "A partir del 15 de marzo de 1965 y por el resto del año académico que concluye

\footnotetext{
35 ARCHIVO HISTÓRICO, Universidad Pedagógica y Tecnológica de Colombia. Actas Consejo Académico, 1963, Tomo I, Oficio del 24 de enero de 1963.
} 
el 31 de enero de 1966, nombrase a la señorita MARÍA TERESA LEÓN LEAL" ${ }^{36}$ (retrato 2), como profesora Auxiliar de la Especialización de Humanidades e Idiomas, de la Facultad de Ciencias de Educación. Con este ingreso, el proceso de vinculación de mujeres como docentes a la UPTC se consolida.

El ingreso de las primeras educadoras a la Universidad Pedagógica y Tecnológica de Colombia coincide con el gobierno del presidente Guillermo León Valencia ${ }^{37}$ (19621966); es importante destacar que la hermana de este fue al Congreso a defender los derechos de la mujer, aunque él estaba en desacuerdo. El orden público tuvo en este gobierno, el segundo del Frente Nacional, especiales significaciones. "La pacificación de la república fue su objetivo principal y, para lograrlo, enfrentó con decisión a los violentos. En este aspecto, un hecho importante ocurrido durante este gobierno fue el movimiento estudiantil de 1965, que puso en aprietos la estabilidad del gobierno, y que condujo a la declaratoria del estado de sitio, y a que el propio presidente Valencia le solicitara al rector de la Universidad de Antioquia su renuncia, como una contribución a los propósitos del gobierno"38. La Universidad Pedagógica y Tecnológica de Colombia vivió también, por esta época, momentos de conflicto interno, una de las razones de la renuncia irrevocable como rector del doctor Azula Barrera y de la primera docente, Lucía Corsi Otálora.

Por otra parte, en este periodo, específicamente en el año 1965, se presenta el mayor número de nombramientos de mujeres: cinco; esto representa un espacio ganado por estas, y hace que cada día se incremente el número de mujeres docentes que ingresan a la Universidad; algo muy importante es que se estabiliza su vinculación, a pesar de ser anuales los nombramientos; ellas son confirmadas cada año y se presenta una continuidad de docentes en razón de su capacidad intelectual, cumplimiento y respeto ante la comunidad académica.

\section{Tendencia de la vinculación de Educadoras a la Universidad Pedagógica y Tecnológica de Colombia}

El inicio de la llegada de mujeres docentes a la Universidad Pedagógica y Tecnológica de Colombia se da en una época en que hay un despertar feminista en todo el mundo y un movimiento de las mujeres por ocupar espacios públicos en un mundo imaginado solo para

\footnotetext{
${ }^{36}$ María Teresa León Leal. Nació en El Cocuy, Boyacá. Realizó sus estudios primarios en su pueblo natal, los secundarios en el Instituto Pedagógico y universitarios en la Universidad Pedagógica Nacional de la ciudad de Bogotá, donde recibió el título en Filología e Idiomas, también realizó estudios de inglés en Estados Unidos e Inglaterra. En historia de vida N ${ }^{\circ} 3$

37 LEÓN VALENCIA, Guillermo: Político caucano (Popayán, 1909 - Nueva York, 1971), presidente de la República durante el periodo 1962-1966. Esta fue una época en que la relación entre los partidos se caracterizó por odios profundos que impedían la comunicación entre los grupos políticos. De aquí que la disposición de Valencia de cubrir «milimétricamente» los puestos públicos se hubiera hecho célebre, y para ocuparlos fueron llamados tanto los conservadores como los liberales, actitud que provocó el agradecimiento de unos y el desconcierto de otros. Extraído él $20 / 01 / 2009$ desde internet (online) desde http:// www.lablaa.org/blaavirtual/biografias/valeleon.htm

${ }^{38}$ Ibídem, p 23.
} 
hombres. Esta tarea no fue fácil; se dudaba de la capacidad de las mujeres, en algunos casos; en otros, no eran autorizadas por sus esposos para que salieran a lo público, y en otras circunstancias se les asignaba carga académica en materias y programas que se consideraban de segunda categoría. "El acceso a la universidad es una de las realizaciones más tardías de las mujeres docentes" ${ }^{\prime 3}$. Pero a pesar de esta situación, lentamente se incorporan, inicialmente en las facultades de educación, posteriormente en las de ingeniería y, poco a poco, se amplía su presencia en la mayoría de facultades y, por ende, de profesiones.

Como se dijo, la presencia de las mujeres docentes en la Universidad Pedagógica y Tecnológica de Colombia se inicia en 1961, con la llegada de la licenciada Lucía Corsi Otálora, a la Escuela de Filología e Idiomas, de la Facultad de Educación. Más tarde, en el año 1965, se vinculan seis profesoras, nuevamente a la Facultad de Educación: tres a la Escuela de Idiomas, dos a la de Biología y Química y una a Psicopedagogía. Posteriormente, en 1968, llega la primera mujer al programa de matemáticas. En estos años continúa a cuenta gotas el ingreso de mujeres, hasta alcanzar un total de 26 profesoras al finalizar la década de los sesenta, en toda la Universidad, cifra mínima, comparada con la de 317 hombres docentes.

\section{María Teresa León Leal: Primera Educadora de Planta en la UPTC}

Dentro del anterior contexto, se presenta la vinculación a la UPTC de María Teresa León Leal, como primera educadora de planta. Hija de profesores, María Teresa realizó sus estudios primarios en El Cocuy, Boyacá, y fue enviada interna a la ciudad de Bogotá a hacer los estudios secundarios en el Instituto Pedagógico Nacional, para luego continuar su formación profesional en la Universidad Pedagógica Nacional de Bogotá, donde se gradúo en Filología e Idiomas; posteriormente perfecciona sus estudios en Estados Unidos e Inglaterra.

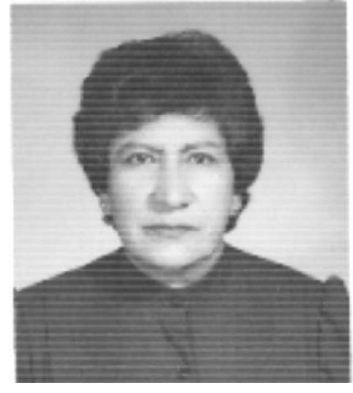

Retrato $\mathbf{N}^{\circ}$ 2. MARÍA TERESA LEÓN LEAL. Primera Educadora de planta Facultad de Educación, 1965. Universidad Pedagógica y Tecnológica de Colombia
El doctor Rafael Bernal Jiménez ${ }^{40}$, entonces rector de la UPTC, la nombra docente de inglés en la Normal de Varones, que era adscrita a la Universidad, y posteriormente, por solicitud expresa del Decano de la Facultad de Educación, doctor Alberto Jiménez, se vincula a dictar inglés técnico en Agronomía; posteriormente pasa a la Escuela de Idiomas.

El inglés de la Escuela de Idiomas se debía enseñar a leer, escribir, escuchar y hablar, en el

\footnotetext{
39 FLECHA GARCÍA, Consuelo. (2008): Mujeres y Universidad. Buenos Aires, p. 39.

40 Ante la renuncia del Dr. Azula, asume la rectoría, en julio de 1963, el Dr. Rafael Bernal Jiménez, quien, además de recibir una universidad en crisis, tuvo que afrontar durante todo su gobierno una serie de manifestaciones y huelgas que también llevaron a su renuncia en el mes de mayo de 1965, al no poder afrontar la crisis financiera, debido a que se mantuvo el mismo presupuesto, no obstante el incremento de estudiantes y profesores.
}

\begin{tabular}{l|l} 
Rhela. Vol. 12. Año 2009, pp. $28-46$
\end{tabular}


inglés técnico de otras escuelas se enseñaba a leer y comprensión de textos, lo consideraban como fácil y algo secundario, es decir, de poca importancia; inicialmente tuve que dictar este inglés técnico, me dijeron que fuera allá que no habia más, pero contesté que a donde fuera lo dictaba. ${ }^{41}$

María Teresa León Leal laboró en la UPTC hasta que salió pensionada, fue maestra de todas las mujeres que ingresaron a la universidad, y debió abrir el camino a todas las mujeres en las diferentes actividades de la vida académica universitaria; ella, con toda su sabiduría e inteligencia, desarrolló toda su actividad igual que los demás docentes, es así que:

Dictaba clases magistrales, con la utilización de tablero, tiza y, en algunas ocasiones, copias en stencil para ejercicios o talleres del inglés. La mayoría había aprendido la metodología para dictar clases en la Universidad Pedagógica de Bogotá y en en el Instiruto Pedagógico, donde obtuve mi formación pedagógica. Preparar clases llevaba mucho tiempo, normalmente lo hacían en la Universidad después y antes de dictar clases, y, en algunas ocasiones, en las noches en las casas, cuando no interfería la familia. ${ }^{42}$

La primera mujer docente que dictó clases en los "cursos de capacitación"43, en los que se centraba la extensión universitaria en ese entonces, fue la maestra María Teresa León (Fonología), los días 22, 23, 24 y 28 de junio y 1, 2, 6, 8, 9, 13, 15 y 16 de julio de 1970 en compañía de varios docentes; estos cursos de actualización y capacitación estaban dirigidos a docentes de diferentes colegios de Boyacá y a docentes de la Universidad, y se realizaban en el periodo de vacaciones; por ejemplo, cursos de idiomas, matemáticas y biología. En 1971, la profesora María Teresa Leon Leal da inicio a la participación de las mujeres en la productividad académica; presenta su primer trabajo de ascenso a la Comisión de Personal Docente. ${ }^{44}$

También es importante destacar que la maestra María Teresa inicia la realización de publicaciones de profesoras de la Facultad de Educación, publicaciones que hacen parte de la riqueza científica de la Biblioteca Central de la UPTC y que son fuente de información para muchas investigaciones. Communicatión aidsin in teaching e Initial teaching alphabet: a new way to teach reading son publicaciones que corresponden a trabajos presentados por las docentes para ascenso de categoría y como ayuda educativa para el desarrollo de sus clases; estas publicaciones se inician diez años después del ingreso real de docentes a la Universidad; por otra parte, son las docentes de la Facultad de Educación pioneras en tener la iniciativa de publicar sus investigaciones.

Realmente, a esta docente le correspondió asumir la responsabilidad de abrir para la mujer todos los espacios de actividad académica en la Universidad; como se mencionó

\footnotetext{
${ }^{41}$ Historia de vida María Teresa León Leal.

42 León Leal, op. cit. pp.3.

42 Ibídem, pp.5.

44 ARCHIVO HISTÓRICO, Universidad Pedagógica y Tecnológica de Colombia Acta $\mathrm{N}^{\circ}$

27 del 25 de octubre de 1971, comisión docente.
} 
anteriormente, la docente Lucía Corsi, que fue la primera en ingresar a la Universidad, para esta época ya se había retirado. Por lo anterior, es meritoria la labor que realizó la docente María Teresa León, quien demostró capacidad y en todo momento respondió a las exigencias del trabajo académico, lo que creó un ambiente favorable al ingreso de otras docentes a la Universidad.

\section{CONCLUSIONES}

La llegada de las primeras educadoras a la Universidad Pedagógica y Tecnológica de Colombia en el año 1961 fue un acontecimiento muy difícil, hasta el punto de llegar a generar paros y protestas; pero a pesar de ello, representa un acontecimiento transcendental en la Universidad Pedagógica y Tecnológica de Colombia, en la medida que abre un espacio profesional para la mujer, nunca imaginado, y que hasta ahora estaba cultural, social y económicamente asignado a los hombres.

El ingreso de la mujer a una institución académica y la realización de un trabajo intelectual en un contexto diseñado mental y físicamente solo para hombres se lograron por la capacidad e inteligencia de estas mujeres, pertenecientes la gran mayoría a una clase social alta, que les permitió asumir con propiedad y mucha responsabilidad esta labor, para lograr reconocimiento y respeto de la comunidad académica, que garantizó el nombramiento de más mujeres docentes en los claustros universitarios.

El perfil profesional de estas docentes corresponde inicialmente a mujeres con estudios secundarios en Escuelas Normales e Institutos Pedagógicos, que les facilitó el ingreso a la Universidad Pedagógica Nacional, a realizar sus estudios de licenciatura en idiomas, lo que posibilitó, posteriormente, la llegada de las primeras docentes de idiomas a la Facultad de Educación. Más tarde, estudiantes de diferentes colegios de Tunja, entre los más destacados, Normal de Varones y Colegio de Boyacá, ingresan a la UPTC para realizar sus carreras profesionales en diferentes áreas, y luego se vinculan como docentes.

Estas primeras educadoras son profesionales de familias destacadas de Boyacá; sus padres, en la mayoría, son Secretarios de Educación, Supervisores de Educación Media, profesores o personas independientes, económica y socialmente sobresalientes. Es admirable la calidad de las docentes de estos primeros años de universidad, debido, entre otros factores, a que estudiaron en los mejores colegios y universidades de la época, tuvieron la oportunidad de capacitarse en el exterior y porque pertenecen a familias importantes; sobresalen por un alto sentido de responsabilidad social y un deseo grande de ser las mejores docentes; hecho que, indudablemente, sirvió de base para la vinculación paulatina de más docentes.

Los nombramientos de docentes son acompañados de varios hechos y costumbres que están presentes en todo acto administrativo, y que algunas veces obligan a expedir una norma para legalizarse y poder funcionar normalmente dentro de una realidad y 
una norma existente. Las docentes de las décadas de los sesenta se incorporan en la medida que presentan sus hojas de vida y cumplen con los perfiles, necesidades y requisitos. Para la década de los setenta ingresan por medio de convocatoria que hace la Universidad, según las necesidades de servicios que presenta el director de Escuela al Consejo Académico y este al Rector; inicialmente presentan hoja de vida y entrevista, y en la medida que crece la Universidad y hay más oferta de profesionales, se hace a través de convocatoria con examen escrito y entrevista.

La mujer docente se incorpora a la UPTC en la década reformista de los sesenta, que "corresponde a la etapa de estabilización, reorganización estructural y académica de una institución únicamente pedagógica a una entidad pedagógica y tecnológica" 45 . A partir de la década de los sesenta se inicia una nueva etapa en la historia de la institución, tendiente a la implementación de nuevas áreas del conocimiento, como la tecnológica, para capacitar técnica e intelectualmente personal especializado.

El impacto social que genera la vinculación de las primeras mujeres docentes a la Universidad Pedagógica y Tecnológica es muy grande, en la medida que abre un nuevo escenario para la mujer, que le exige prepararse y demostrar sus capacidades, para poder desempeñar una profesión con mucha calidad y responsabilidad, como lo hicieron ellas en su momento y, así, contribuir con el progreso de la institución.

Por último, la educadora María Teresa León Leal fue la primera mujer maestra de planta en la UPTC; con su trabajo académico demostró que las mujeres tienen iguales capacidades que los hombres para desarrollar el trabajo científico en la universidad, que, por lo tanto, el conocimiento es universal y que no deben presentarse ninguna exclusión.

${ }^{45}$ OCAMPO LÓPEZ, Javier. Op. Cit, p. 233. 


\section{FUENTES}

ARCHIVO

ARCHIVO HISTÓRICO, Universidad Pedagógica y Tecnológica de Colombia, Acta No 7 de 1954, Consejo Directivo, legajo $\mathrm{N}^{\circ} 2$.

ARCHIVO HISTÓRICO, Universidad Pedagógica y Tecnológica de Colombia, Asuntos varios rectoría, junio 1955.

ARCHIVO HISTÓRICO, Universidad Pedagógica y Tecnológica de Colombia, Acta $\mathrm{N}^{\circ} 5$. Consejo Directivo Carpeta $\mathrm{N}^{\circ} 6$ de 22 de marzo de 1957.

ARCHIVO HISTÓRICO, Universidad Pedagógica y Tecnológica de Colombia, Asuntos varios rectoría. Acta Consejo Directivo No 4 de 1957.

ARCHIVO HISTÓRICO, Universidad Pedagógica y Tecnológica de Colombia, 1960-1980.

\section{HISTORIAS DE VIDA}

Historia de vida N. ${ }^{\circ}$ 3. María Teresa León Leal

Historia de vida N. ${ }^{\circ} 4$. Lucía Corsi Otálora

Historia de Vida N. ${ }^{\circ}$ 8. Carmen Mariño

Historia de Vida N. ${ }^{\circ} 9$. Cecilia Malagón

Historia de Vida N. ${ }^{\circ}$ 10. Bertha Iris Rodríguez de Buitrago

\section{SELECCIÓN BIBLIOGRÁFICA}

ACTIS, Adriana. (1995): La mujer en la universidad, Lerner, Buenos Aires.

ACKER, Sandra. (2003): Género y Educación, Madrid, Narcea, S. A de ediciones.

ARCHILA, Mauricio. (2001): Movimientos Sociales, Estado y Democracia en Colombia. Bogotá: Litocamargo Ltda.

BONDER, Gloria. (1994): “Género y Educación”, en Revista Iberoamericana de la educación, No 6, Argentina.

BONILLA, Elsy. (1993): "La mujer Colombiana en la universidad," en Centro de Estudios sobre Desarrollo Económico, CEDE, Universidad de los Andes.

BRITTAN, A. (1989): Masculinity and power, Nueva York, Brasil Blackwell.

BROOKS, Ann. (1997): Academic women, Bckingham, Open University Press.

CORREA OLARTE, María Eugenia. (2005): La Feminización de la educación superior, Bogotá, Tercer mundo editores.

CASTRO BARBOSA, Sol Mercedes. (2008): La mujer docente universitaria en Colombia. Proceso de formación académica e inserción laboral en la universidad (1935-1975), Tunja, Tesis de grado para optar el título de Doctor en Ciencias de la Educación, Universidad Pedagógica y Tecnológica de Colombia, RUDECOLOMBIA.

CÓRDOBA CURREA, Claudia Calorina. (2007): Estudios de género en el Departamento Nacional de Estadísticas - DANE, Bogotá, Universidad Javeriana. 
CLARK, Veve. (1996): Antifeminism in the Academy, Nueva Yorrk, Routledge.

GUIL BOZAL, Ana. (2007): "Docentes e Investigadoras en las Universidades Españolas," en Revista de Investigación Educativa. Vol.25-1.

. (2007): La Situación de las mujeres en las universidades públicas andaluzas, Consejo Económico y social de Andalucía, Publicaciones CESA.

HELG, Aline. (1987): La educación en Colombia.1918-1957, Bogotá, Presencia.

MUNÉVAR M. Dora Inés. (2004): Poder y género en el trabajo académico. Universidad Nacional de Colombia. Facultad de Medicina, Bogotá, Unibiblos.

OCAMPO LÓPEZ, Javier. (1978): Educación, Humanismo y Ciencia, Tunja, Ediciones La Rana y el Águila.

PARRA BÁEZ, Lina Adriana. (2006): La formación de maestras universitarias colombianas entre 1950-1960, Tunja, Tesis de grado para optar el título de doctor en Ciencias de la Educación. Universidad Pedagógica y Tecnológica de Colombia - RUDECOLOMBIA.

PIÑERES DE LA OSSA, Dora. (2004): Historia de la Universidad de Cartagena. Reforma y Modernidad 1920 - 1946, Tunja, Tesis de grado, Doctorado en Ciencias de la Educación. Universidad Pedagógica y Tecnológica de Colombia - RUDECOLOMBIA.

SOTO ARANGO, Diana. (1992): Resumen analítico en la historia de la educación Latinoamérica, Bogotá, Universidad Pedagógica Nacional.

. (2005). “Aproximación histórica a la universidad colombiana”, en Revista Historia de la Educación Latinoamericana $\mathrm{N}^{\circ}$ 7, Tunja, Universidad Pedagógica y Tecnológica de Colombia- RUDECOLOMBIA.

. (2007): "La investigación y la innovación en los programas de historia y ciencias sociales en Colombia. Una propuesta desde el proyecto 6 × 4", en Revista Historia de la Educación Latinoamericana $\mathrm{N}^{\circ}$ 9, Tunja, Universidad Pedagógica y Tecnológica de ColombiaRUDECOLOMBIA.

SCHIEBINGER, Londa. (1992): ¿Tiene sexo la mente?, Valencia España, Instituto de la mujer, ediciones cátedra.

HAMÓN NARANJO, Ana. (2009): “Abriendo Nuevos Espacios Académicos y Científicos: Primeras Educadoras en la Universidad Pedagógica y Tecnológica de Colombia" en Revista Historia de la Educación Latinoamericana Vol. 13, Tunja, Universidad Pedagógica y Tecnológica de Colombia, RUDECOLOMBIA, pp. $28-46$. 